$16^{\text {th }}$ International Congress of Metrology, $13002(2013)$

DOI: $10.1051 /$ metrology/201313002

(C) Owned by the authors, published by EDP Sciences, 2013

\title{
Deux ans d'expérience du club nanoMétrologie
}

\author{
F. Piquemal ${ }^{1}$, K. Aguir ${ }^{2}$, D. Bernard ${ }^{3}$, V. Cappronnier ${ }^{1}$, J. Carimalo ${ }^{4}$, D. Chambonnet ${ }^{5}$, Y. De Wilde ${ }^{6}$, S. Ducourtieux ${ }^{1}$,

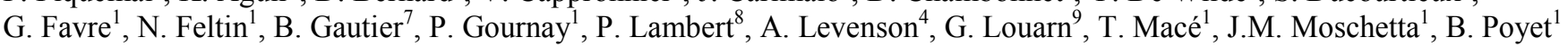 \\ ${ }^{1}$ LNE, 29 avenue Roger Hennequin, 78197 Trappes Cedex, France \\ ${ }^{2}$ Aix-Marseille Université - IM2NP, St. Jérôme, 13397 Marseille, France \\ ${ }^{3}$ Arkema France, 420 rue d'Estienne d'Orves 92705 Colombes Cedex, France \\ ${ }^{4}$ NanoSciences France (C'Nano), CNRS/LPN, Route de Nozay - 91460 Marcoussis - France \\ ${ }^{5} 3 \mathrm{~S}$ Photonics, Route de Villejust, 91625 Nozay, France \\ ${ }^{6}$ Institut Langevin, ESPCI/ParisTech, 10 rue Vauquelin, 75005 Paris, France \\ ${ }^{7}$ INL/INSA Lyon, 7, avenue Capelle, 69621 VILLEURBANNE Cedex, France \\ ${ }^{8}$ Institut Jean Lamour, CNRS/Université de Lorraine, BP 70239, 54506 Vandoeuvre les Nancy, France \\ ${ }^{9}$ Institut des Matériaux Jean Rouxel - IMN, BP 32229 ; 2 rue de la Houssinière, 44322, Nantes Cedex 3, France
}

\begin{abstract}
Résumé. Créé en 2011 conjointement par le LNE et NanoSciences France (réseau C'Nano), le club nanoMétrologie a pour objectif de rassembler industriels, secteur académique et agences gouvernementales au sein d'un véritable réseau afin de partager des problématiques métrologiques dans tous les domaines que recouvrent les nanosciences et les nanotechnologies. 260 adhérents sont actuellement recensés dont un tiers provient du secteur industriel (producteurs, entreprises de transformation et d'intégration, fabricants d'instruments). Les principaux résultats obtenus par les groupes de travail du club et qui marquent ces deux premières années seront présentés et quelques perspectives d'actions seront données.
\end{abstract}

\section{Introduction}

Depuis plus d'une décennie, les nanotechnologies couvrent un domaine tout à fait nouveau pour lequel les fabricants, les agences gouvernementales et les citoyens ont des attentes très fortes. En effet, les nanotechnologies offrent un nombre toujours croissant d'applications potentielles dans tous les secteurs clés de l'industrie [1]:

- l'énergie (cellules photovoltaïques avancées, éclairage à diodes électroluminescentes, dispositifs de stockage, etc) ; - le transport (pneumatiques, membranes pour piles à combustible économiques, surfaces vitrées intelligentes, etc) ;

- la construction et le bâtiment (peintures réfléchissantes solaires autonettoyantes et antimicrobiennes, béton, etc) ;

- le médical et l'industrie pharmaceutique (implants et revêtements pour la médecine régénérative, biocapteurs, etc) ;

- les technologies de l'information et de la communication (électroniques de grande consommation, lasers, écrans flexibles, impression à jet d'encre, etc) ;

- le textile dans des applications de sécurité et de protection individuelle, médicales ou sportives ;

- les biens de consommation en cosmétique (hydratants, maquillage, protection solaire, etc) ou en produits d'entretien ménagers (nettoyants, savon, etc) ;

- l'emballage (alimentation, boisson, médicaments, vêtements, etc).
Certaines de ces applications recouvrent également le domaine de la santé (imagerie par médication vectorielle, thérapie) et de l'environnement (énergie verte, traitement durable de l'eau). Les nanomatériaux, produits ou procédés les mettant en œuvre existent déjà, mais les moyens de mesurer leurs propriétés sont encore à leurs balbutiements. Il est souvent difficile de mettre en place une instrumentation capable de mesurer la taille, la forme et les propriétés physico-chimiques des nano-objets avec les incertitudes requises. Par ailleurs, les processus industriels impliquent une gestion de l'assurance de la qualité qui s'appuie sur des outils fiables pour lesquels la métrologie détient toujours un rôle clé.

Le soutien aux industriels dans le domaine en pleine croissance des nanotechnologies et l'analyse du rapport bénéfices/risques des nanomatériaux exigent ainsi le développement d'une métrologie spécifique aux dispositifs nanométriques : la nanométrologie [2-5]. Il ne s'agit pas seulement d'étendre la métrologie dimensionnelle à l'échelle nanométrique mais de développer toute une métrologie au service des nanosciences et des nanotechnologies. Elle se distingue alors de la métrologie traditionnelle par son aspect multidisciplinaire mais aussi par le fait qu'elle nécessite l'émergence de nouveaux concepts (métrologie hybride, fusion de données, etc).

En parallèle, le développement des nanomatériaux pose la question de leur réglementation et des risques possibles en matière de santé et d'environnement. Dans le cadre de 
la loi dite «Grenelle 2 », un décret sur une déclaration annuelle obligatoire des produits contenant des substances à l'état nanoparticulaire a été publié en février 2012 et prend effet à partir de janvier 2013. Cependant, tous les rapports publiés par les agences gouvernementales et les organisations de normalisation [6-9] soulignent le manque d'outils, de matériaux de référence et de méthodes qui permettraient d'établir la traçabilité des mesures, d'améliorer ainsi leurs comparaisons et de faciliter in fine l'application du décret. Cela soulève une seconde différence par rapport aux secteurs traditionnels.

Pour ces deux raisons principales qui pourraient expliquer l'absence actuelle d'un véritable secteur industriel des nanotechnologies en France (pas de fédération ni de syndicat), le LNE et NanoSciences France, un réseau interdisciplinaire initié par le CNRS, le CEA et le Ministère de l'Enseignement Supérieur et de la Recherche, créent en 2011, le Club nanoMétrologie (fig.1).

Figure 1 Logo du club

\section{Club nanoMétrolagie}

Son objectif est de rassembler industriels, secteur académique et agences gouvernementales au sein d'un véritable réseau pour :

- dresser un état des lieux en matière d'instruments disponibles sur le territoire national, d'étalons et de chaînes de traçabilité pour toutes les grandeurs mesurées dans le domaine des nanosciences et des nanotechnologies; - recueillir et préciser les besoins en nanométrologie venant directement des industriels ;

- mettre en commun des problématiques métrologiques y compris celles relatives aux aspects toxicologiques et écotoxicologiques des nanomatériaux ;

- venir en soutien aux travaux de normalisation ;

- assurer la diffusion de connaissances métrologiques au monde industriel et académique ;

- aider à la réflexion pour l'élaboration de programmes de recherche. Dans ce cadre là, le club fait également office d'incubateur de projets partenariaux.

\section{Organisation du club}

Le pilotage du club est assuré par un comité exécutif constitué de membres du LNE et de NanoSciences France ainsi que de deux industriels. Il est soutenu par un comité scientifique et technique qui encadre les travaux du club au sein de groupes de travail (GT). Ces derniers ont pour objectifs de dresser un état des lieux sur les besoins métrologiques dans des thématiques spécifiques afin d'établir des priorités. Ils sont actuellement au nombre de quatre et traitent les aspects suivants :

- Les besoins de mesures dans le domaine de la santé et de l'environnement (GT1) ;

- La traçabilité des mesures à l'échelle nanométrique : besoins en matériaux de référence, étalons, chaîne de traçabilité, méthodologie, bilan d'incertitudes (GT2) ;

- Les besoins en instruments de mesure spécifiques aux nanotechnologies (GT3) ;
- La modélisation et la simulation en lien avec la nanométrologie (GT4).

Les groupes de travail sont organisés de façon à équilibrer la représentativité du secteur industriel, académique et des agences gouvernementales, pour prendre en compte toutes les facettes des nanosciences et des nanotechnologies.

Par ailleurs, depuis septembre 2011 les adhérents du Club bénéficient d'un accès à une plate-forme collaborative en ligne qui diffuse des informations générales, des bibliographies, des documents de normalisation ainsi que des rapports synthétisant les travaux des groupes de travail et des présentations effectuées lors des diverses réunions organisées au sein du club. Un forum de discussion a été mis en place pour favoriser les échanges entre adhérents.

Un site internet [10] a été créé et permet une meilleure visibilité sur les objectifs et les activités du club. Un journal électronique de type « Newsletter » disponible sur le site publie les articles soumis uniquement par les membres du club (résultats scientifiques et techniques, nouvelles générales : organisation, infrastructure, etc).

Enfin, pour jouer pleinement son rôle de réseau, il est rappelé que le club est largement ouvert à toutes les personnes intéressées par les thématiques proposées. Les inscriptions se font via le site internet. Le club organise annuellement en fin d'année, une journée ouverte (adhérents et non adhérents) «Les rencontres annuelles en nanométrologie » lors de laquelle les résultats des travaux de chaque groupe de travail sont restitués et des interventions sur des aspects scientifiques, industriels ou politiques phares sont organisées. Pour l'exercice 2013, elles auront lieu au LNE-Paris le 11 décembre.

\section{Statistiques}

249 adhérents ont été recensés à mi année 2013 (contre 154 à fin 2011 soit une progression de $70 \%$ ), avec une proportion inchangée de plus d'un tiers provenant du secteur industriel (fig.2).

Figure 2 Répartition des adhérents du club en fonction de leur secteur d'activité

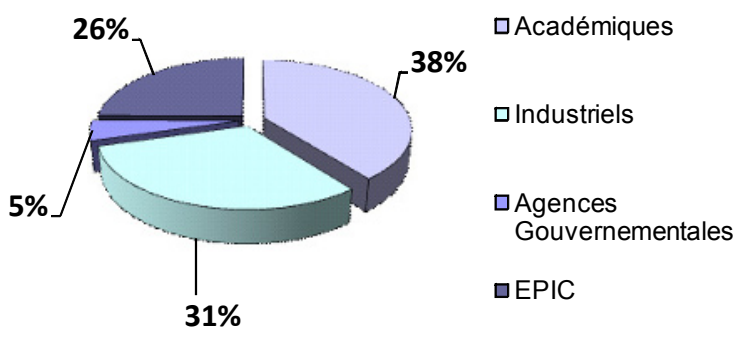

$\mathrm{Au}$ sein du club, le secteur industriel est représenté par plus de 40 entreprises réparties en proportion égale entre PME et grandes compagnies. Le principal secteur d'activité des PME est l'instrumentation (électronique, optique, mécanique, électrique et magnétique, gaz, technologie du vide, logiciel), secteur dans lequel les PME interviennent majoritairement en tant que fabricant, distributeur ou assistant en ingénierie. Parmi les quelques PME restantes, on compte un prestataire d'analyse de surface de matériaux, deux sociétés commerciales de produits chimiques et de produits électriques et une société 
biopharmaceutique (technologie innovante dans l'administration de médicament). Le secteur d'activités des grandes entreprises couvre l'instrumentation, la conception et le développement de composants (électroniques, optiques, mécaniques) et d'équipements (matériel en environnement contrôlé, etc), l'élaboration de produits chimiques (colles, etc) et de matériaux, mais également de combustibles, métaux et autres minéraux. Certaines d'entre elles interviennent au sein du club par l'intermédiaire de leurs centres de recherche et développement.

Les activités de toutes ces entreprises industrielles s'inscrivent dans les filières technologiques suivantes: micro, nano et opto-électronique, chimie, biologie, santé, énergie, environnement, bâtiment, textile et papier, aérospatial, automobile, matériaux et procédés, défense et sécurité.

A l'issue de la première année d'existence du club, un questionnaire a été diffusé auprès des adhérents pour mieux définir les attentes que suscite le club et ainsi mieux préciser les objectifs. La synthèse des résultats de l'enquête (72 répondants, $57 \%$ provenant d'organismes publics, $43 \%$ du secteur privé, issus dans $55 \%$ des cas du domaine de la $\mathrm{R} \& \mathrm{D}$, les autres domaines d'activité les plus représentatifs et de poids équivalent (8\%) étant le service, la valorisation, les essais, la production et la distribution), montre principalement que le type d'amélioration attendu par les participants au travers du club concerne la compétitivité, le produit et le service avec un souhait unanime $\mathrm{du}$ rapprochement entre la recherche et l'industrie. Les trois raisons principales qui ont motivé leur adhésion sont (i) l'intégration à un réseau de métrologie/nanométrologie, (ii) le développement de compétences techniques complémentaires et (iii) la découverte de nouveaux débouchés (clients, nouvelles technologies / techniques).

Sur le type de participation au club, la majorité des répondants se sont déclarés vouloir participer aux réunions, consulter les documents issus des GT, être concernés par des propositions de programmes, des actions et être à la recherche de partenariat. Les réponses ont enfin permis de disposer d'une photographie du réseau entre les deux communautés industrielle et académique qui sert de point de référence pour le club.

$\mathrm{Ce}$ questionnaire est désormais envoyé systématiquement à tout nouvel inscrit afin d'effectuer une étude dynamique des besoins des adhérents.

\section{Résultats des GT}

Les travaux réalisés sur les deux premières années ont abouti aux résultats résumés ci-après. Ils ont été obtenus majoritairement à l'issue de journées thématiques intergroupes de travail (comptes rendus des réunions disponibles sur la plateforme collaborative):

- Un état des lieux sur la mesure dimensionnelle des nanoparticules en solution ou déposées sur substrat avec un focus sur l'instrumentation, l'échantillonnage, l'étalonnage et la détermination des bilans d'incertitudes ;

- Une vue d'ensemble sur la métrologie des nanoaérosols : cette métrologie est déterminante pour établir une traçabilité des mesures sur site et pour mieux étudier l'impact sanitaire des nanoparticules. Le manque d'un nano-aérosol de référence a largement été mentionné et un cahier des charges est en cours d'écriture pour élaborer un tel étalon;

- L'identification de besoins criants en matière de métrologie pour la mesure de rugosité (fig.3) et de couches minces par ellipsométrie et profilométrie optique (sujet touchant le domaine de la microélectronique et de l'optique) : les besoins s'expriment en termes d'étalons de référence pour des comparaisons in situ, des moyens d'étalonnage d'ellipsomètres à mettre en œuvre en France, des étalons de transfert à base de couches minces et des étalons de valeurs d'indice avec une exactitude de l'ordre de $10^{-4}$. Il a été décidé de poursuivre le sujet par un recensement exhaustif des étalons de référence pour la mesure de couches minces par ellipsométrie et par la définition d'un cahier des charges sur les différents étalons afin de construire un projet de R\&D qui comprendra 9 partenaires dont 5 industriels ;

Figure 3 Mesure de rugosité sur un film d'or par microscope à force atomique (rugosité arithmétique $R a=2,3 \mathrm{~nm}$ mesurée sur une image de $10 \mathrm{~nm} \times 10 \mathrm{~nm})$.

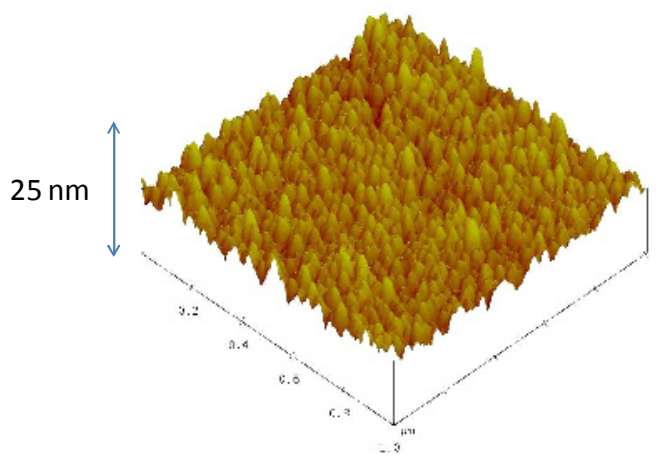

- Une revue des besoins en métrologie des grandeurs électriques à l'échelle nanométrique : ils recouvrent les techniques de mesure électrique à sondes locales (courant, impédances, potentiel de surface) et les aspects normatifs associés, les moyens de mesure en microélectronique en milieu industriel, la mesure du potentiel zêta concernant les nanoparticules et la modélisation des phénomènes de transport dans les nanodispositifs. Les besoins se répartissent en deux catégories: (i) les pointes et les interactions pointe - échantillon, (ii) les mesures et leur interprétation ;

- Le recensement des différentes techniques et moyens de mesures disponibles en France pour la nanométrologie : un fichier a été constitué collaborativement où figurent aujourd'hui 150 instruments et pour chacun d'entre eux, les informations sur une quarantaine de paramètres (les grandeurs accessibles par la technique de mesure, la gamme de mesure, les points faibles et les points forts, les limitations, les conditions de mesure, la traçabilité et le mode de la mesure (destructive, intrusive) ainsi qu'un contact expert au sein du club. Ce fichier est régulièrement mis à jour. Les informations contenues dans ce document peuvent être disponibles gracieusement sur simple demande;

- Une première enquête menée au sein du club a permis d'identifier deux domaines dans lesquels les attentes en matière de modélisation et simulation sont les plus précises en matière de mesure pour les nanotechnologies : 
le domaine regroupant les activités relatives à la micro et nano électronique et aux micro/nanosystèmes, et le domaine de la caractérisation physicochimique des nanomatériaux (nouvelles applications et analyse de risques en toxicité et écotoxicité).

\section{Conclusion et perspectives}

$\mathrm{Au}$ cours de ses deux premières années d'expérience, le Club nanoMétrologie a montré son fort potentiel dans l'établissement d'une véritable passerelle entre les secteurs industriel et académique pour faire face aux enjeux métrologiques que soulèvent les nanosciences et les nanotechnologies. Son mode de fonctionnement en réseau avec la mise en place d'une plateforme collaborative, un site internet, l'organisation de journées thématiques ... permet une mise en contact efficace des participants et l'émergence de projets communs. Le Club nanoMétrologie a également tenu son rôle de diffuseur de connaissances vers le monde industriel (voir par exemple [11]).

Dans le cadre des actions menées au sein des différents groupes de travail, les problématiques telles que la métrologie des nanoparticules (en solution, déposées sur substrat ou en aérosol) et la nanométrologie dédiée à la micro et opto-électonique sur des grandeurs dimensionnelles et électriques montrent des besoins précis et pour certains urgents en termes d'étalons et de moyens d'étalonnage. De plus, un recensement important des instruments utilisés sur le plan national à des fins nanométrologiques a été réalisé. Tout adhérent a la possibilité de disposer, sur simple demande, des informations sur tel ou tel instrument mais aussi des noms d'utilisateurs experts. Nous reviendrons plus en détail sur ces travaux lors du congrès.

En termes de perspective à court terme, de nouveaux sujets nanométrologiques vont être traités dans le cadre de journées thématiques spécifiques. Il en est ainsi de la nanotopographie (nanométrologie dimensionnelle sur de grandes surfaces), des mesures locales de propriétés thermiques, de grandeurs magnétiques, de flux optique, de la mesure de propriétés mécaniques sur des nanosystèmes simples (nanoparticules sur une surface par exemple)...

Par ailleurs, pour faire suite à une forte demande exprimée par des adhérents, deux comparaisons interlaboratoires ont été récemment proposées au sein du club et démarreront fin 2013 ou début 2014 :
- La première concerne la mesure dimensionnelle d'étalons ou de structures de référence (par exemple un réseau bidimensionnel) dédié à la microscopie champ proche et plus particulièrement à la microscopie à force atomique (AFM). En fonction du choix porté sur l'étalon "voyageur», la comparaison pourrait également concerner d'autres techniques telles que la microscopie électronique (SEM). Le but principal de cette comparaison est de fournir à chaque participant une idée de l'état d'étalonnage de son équipement.

- Dans un même esprit, la seconde comparaison a trait aux mesures dimensionnelles de nanoparticules. Ce travail permettra aussi de comparer les mesures provenant de différentes méthodes fondées sur des principes physiques différents. La restitution des résultats sera suivie d'une discussion sur les protocoles utilisés. Plusieurs types de nanoparticules ont d'ores et déjà été choisis et serviront de matériaux de référence ( $\mathrm{NP}$ de $\mathrm{SiO}_{2}$, d'Au et d'hématite) et sept techniques ont été retenues pour les mesures dimensionnelles (TEM, SEM, AFM, DLS, BET dans le cas de NP sphériques, XRD et FFF).

\section{Références}

[1] Nanofutures, "Integrated Research and Industrial Roadmap for European Nanotechnology”, 2012 http://www.nanofutures.info/

[2] R. Bogue, Sensor Rev. Vol. 27, 2007

[3] Co-nanomet project output, www.co-nanomet.eu

[4] International Technology Roadmap for semiconductors, www.itrs.net

[5] R.K. Leach et al., Nanotechnology, Vol.22, 2011

[6] Rapport d'expertise collective, AFSSET, « Les nanomatériaux - Effets sur la santé de l'homme et sur l'environnement », juillet 2006

[7] Rapport d'expertise collective, AFSSET, «Evaluation des risques liés aux nanomatériaux pour la population générale et pour l'environnement », mars 2010

[8] Rapport NanoDM, AFFSAPS : évaluation biologique des dispositifs médicaux contenant des nanomatériaux, février 2011

[9] « Scientific opinion: the potential risks arising from nanoscience and nanotechnologies on food and feed safety», The EFSA Journal, Vol. 959, 2009

[10] www.club-nanometrologie.fr

[11] « Nanométrologie », Spectra Analyse, numéro spécial à paraître en 2013 